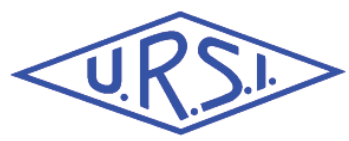

\title{
2-Dimensional sub-atomic localization of Rb Rydberg atoms for SI traceable E-field metrology
}

\author{
Satya K. Dubey* ${ }^{(1)}$ and Harish S. Rawat ${ }^{(1,2)}$ \\ (1) CSIR-National Physical Laboratory (NPL), New Delhi, India \\ (2) Academy of Scientific and Innovative Research (AcSIR), CSIR-NPL, India, http://acsir.res.in
}

\begin{abstract}
In this work, Electromagnetically Induced Transparency (EIT) has been studied in a RF-driven four-level ladder ${ }^{85} \mathrm{Rb}$ atomic model. $\mathrm{Rb}$ atomic cell is simultaneously driven by three fields; two lasers, namely control and probe and one microwave. This spectroscopic technique of exposing atomic vapors to three energies may get established as a quantum primary standard for the RF electric-field amplitude metrology due to its dependency only on universal Plank's constant. It is observed that when right resonant frequency is achieved the absorption spectrum gives a transition to EIT by inducing a window in middle of the curve cancelling the absorption. In addition, two-dimensional localization of an atom is also studied in various field scenarios. The maxima of absorption spectrum can be modified by making slight modifications in the electromagnetic fields driving the system. Next step in the metrology would be of measuring vector E-field for which finding the position of an atom may play a crucial role. This may add one extra degree of freedom in the evaluation of SI traceable E-field.
\end{abstract}

\section{Introduction}

Ever since the invention of laser, atom-light interaction has been a topic of great interest among the physicists. Over the last few decades, ample amount of work has been done throughout the globe on theoretical and experimental analysis of coherences inside atomic gas of rubidium isotope interacting with two lasers. Several groups have successfully progressed to utilize the characteristics of coherences and interferences inside the atoms into a number of applications. Optical response is modified by lasers tuned to atomic states and a number of quantum optic phenomena are recognized such as coherent population trapping [1], Fano effect [2], optical bistability [3], subluminal and superluminal light propagation [4], Electromagnetically Induced Transparency (EIT) [5,6], Autler-Townes splitting [7]. In recent past, the study of atom-photon interaction has been accepted as primary standard for several parameters like length[8], time [8], and frequency [8] because of the dependency only on the universal constants. In view of making all the measurements SI traceable, this technique showed potential possibility of getting accepted as primary standard for radio-frequency E-field measurement [9-11].

In the field of atom-light interaction in quantum mechanics, Heisenberg's uncertainty principle states that the momentum which a photon can transfer to an atom governs the precision of finding the atom. Heisenberg microscope confirms the fact that position of an atom cannot be determined more precisely than the half wavelength of the exciting field. Thus fundamentally an atom cannot be localized within the distances beyond half wavelength of the exciting energy. Recently, some of the research groups have showed that the positions of atoms can be observed more precisely beyond the theoretical limit as well [12-14]. A variety of analytical models has been presented for 1, 2 and 3-Dimensional atom localization [15-17]. Ding et al [15] studied the atomlocalization in the system driven by RF and optical fields and concluded that localization of atoms can be improved by controlling the parameters governing quantum coherence and interference inside the system.

This possibility of finding the atom with high-precision due to the novel techniques may pave the way for vector E-field measurement in the field of quantum metrology. If we are able to locate and change the position of an atom, at will, within the wavelength of the RF field by varying the parameters we can control, we may give the direction vector of the E-field interacting with the atomic vapors inside the atomic cell.

A theoretical background on rubidium atoms interacting with three energies instead of two is presented in this article. In the spectroscopic technique discussed in this work, the $\mathrm{Rb}$ vapour cell is a potential candidate for the standard probe for Electric-field sensing in $1 \mathrm{GHz}$ to 1 $\mathrm{THz}$ range. Also, an initial analytical investigation results are reported about the changes in the absorption spectrum when studied in different combination of standing waves.

\section{Model and Equations}

The time evolution of the atomic model is studied by deriving optical-Bloch equations. The lasers excites the atom from its ground states to the Rydberg states i.e. probe beam couples states $|1\rangle$ and $|2\rangle$, and the states $|2\rangle$ and $|3\rangle$ are driven by a strong control laser with Rabi frequency $2 \Omega_{c}$, shown in Fig. 1 . The third driving field, a 
RF energy with Rabi frequency $2 \Omega_{r f}$ couples the unstable states $|3\rangle$ and $|4\rangle$.

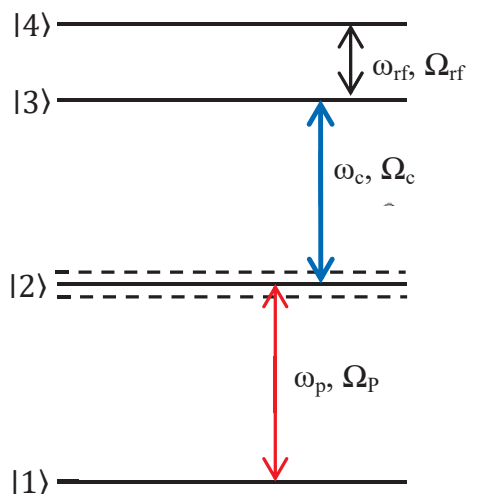

Figure 1 Schematic energy level scheme for ${ }^{87} \mathrm{Rb}$ atom. $\omega_{p}, \omega_{c}$, and $\omega_{r f}$ are the frequencies of Probe, Control and RF energies with $\Omega_{p}, \Omega_{c}$, and $\Omega_{r f}$ as their respective half-Rabi frequencies.

Note that for different values of RF strength the nomenclature of fourth Rydberg state changes every time as Rydberg states are separated by very fine energy margin. Further, RF field cannot effect the transitions between the bare states $|1\rangle$ and $|2\rangle$ as a relatively high energy is required to excite the atom from ground state to its excited state (energy of the order of optical frequencies). The equations obtained by density matrix approach are given as

$$
\begin{gathered}
i \dot{\rho}_{11}=i \Gamma_{12} \rho_{22}-\Omega_{p} \rho_{12}+\Omega_{c}^{*} \rho_{21} \\
i \dot{\rho}_{22}=i \Gamma_{23} \rho_{33}-i \Gamma_{12} \rho_{22}-\Omega_{c} \rho_{23}+\Omega_{c}^{*} \rho_{32} \\
+\Omega_{p} \rho_{12}-\Omega_{p}^{*} \rho_{21} \\
i \dot{\rho}_{21}=t_{2} \rho_{21}+\Omega_{p}\left(\rho_{22}-\rho_{11}\right)+\Omega_{c} \rho_{31} \\
i \dot{\rho}_{31}=t_{3} \rho_{31}+\Omega_{r f} \rho_{41}+\Omega_{c} \rho_{21}-\Omega_{p}^{*} \rho_{32} \\
i \dot{\rho}_{41}=t_{4} \rho_{41}+\Omega_{r f} \rho_{31}-\Omega_{p}^{*} \rho_{42}
\end{gathered}
$$

The set of equations are solved by applying the steady state and weak probe conditions. The off-diagonal element corresponding to the probe absorption can be obtained as

$$
\rho_{21}=\Omega_{p} \frac{\left(\Omega_{r f}^{2}-t_{3} t_{4}\right)}{\Omega_{r f}^{2} t_{2}-t_{2} t_{3} t_{4}+\Omega_{c}^{2} t_{4}} .
$$

By following the method discussed in [18], the dispersion relation $K(\omega)$ for the system can be written as
$K(\omega)$

$=\frac{\omega}{c}-k_{12} \sqrt{\pi} \Omega_{p} \frac{\Omega_{r f}^{2}-\left(\omega+t_{3}\right)\left(\omega+t_{4}\right)}{\left(\omega-\omega_{1}\right)\left(\omega-\omega_{2}\right)\left(\omega-\omega_{3}\right)}$.

The position of the atom passing through the standing wave fields can be understood by the monitoring the susceptibility of the system for the probe laser. The susceptibility can be determined by the off diagonal element $\rho_{21}$ and written as [15]

$$
\chi=\frac{N \mu_{10}}{\varepsilon_{0} E_{p}} K_{\omega}
$$

where $N$ is the atom number density in the medium, $\mu_{10}$ is the atomic dipole moment, $E_{p}$ is the amplitude of the RFfield, and $\varepsilon_{0}$ is the free space permittivity.

\section{Results and Discussions}

The linear response of an atom to resonant light is described by the susceptibility $\chi$. The real part of $\chi$ gives us the refractive index of the medium, while the imaginary part gives the dissipation of field in the medium. The probe absorption in the medium depends on the probe and pump frequencies. Electromagnetically Induced Transparency (EIT) can be observed in the medium only when both the lasers are in resonance with the transition states. Figure 1 represents the induction of the transparent window in the absorption spectrum when the resonant conditions are matched. Red curve shows the case when the control laser frequency is not perfectly matched while, the blue curve represents the transition from perfect absorption to EIT when control Rabi frequency is matched.

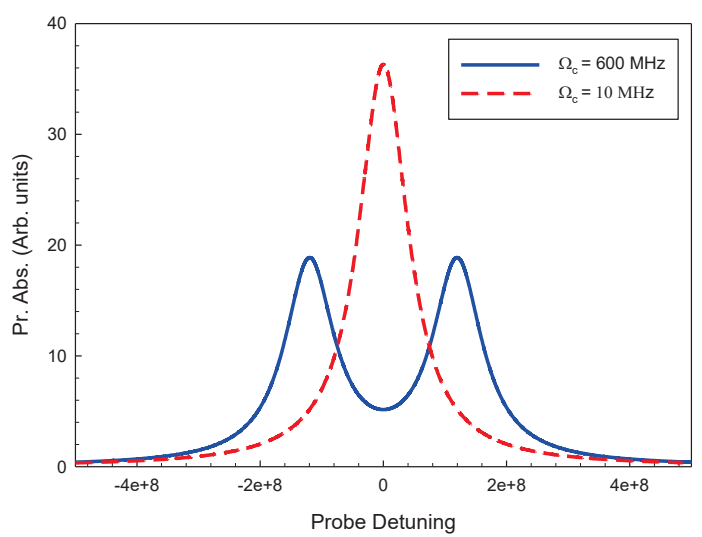

Figure 2 Simulated absorption of probe laser for different values of Rabi frequency of pump laser. Red curve presents the case when Rabi frequency of pump laser is $10 \mathrm{MHz}$ and $600 \mathrm{MHz}$.

Next, the behavior of two-dimensional atom localization is studied using the density matrix approach for different cases and are discussed in this section. The localization 
pattern presented in Fig. 3 shows the wave-like pattern showing the finding probability of the atom is distributed along an $\mathrm{y}$-axis when both control and RF fields are considered as standing waves i.e. $\Omega_{c x}=\Omega_{c} \sin k 1 x$ and $\Omega_{r f y}=\Omega_{r f} \sin k 2 y$. The other system parameters are $\Delta_{p}=1 \mathrm{MHz}, \Delta_{c}=\Delta_{r f}=0, \Omega_{p}=100 \mathrm{MHZ}, \Omega_{c}=$ $400 \mathrm{MHz}, \Omega_{r f}=200 \mathrm{MHz}$.

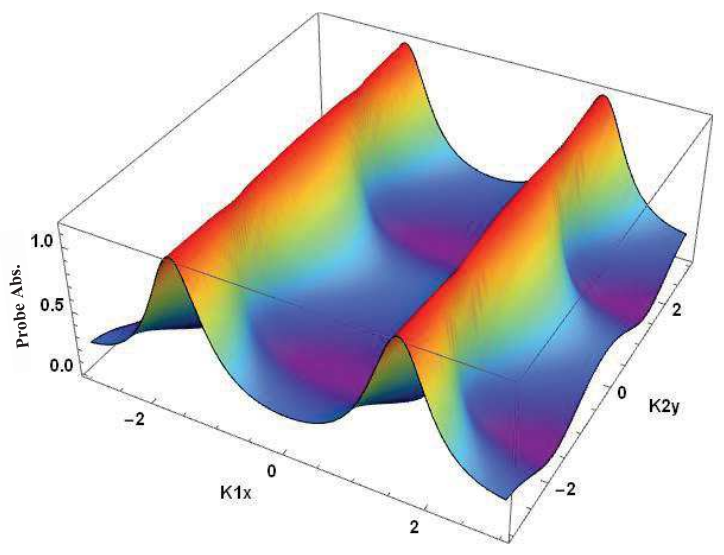

Figure 3 Probe absorption as a function of wave vectors $\mathrm{k}_{1} \mathrm{x}$ and $\mathrm{k}_{2} \mathrm{y}$ reflecting the position probability as wave like structures in the $\mathrm{x}$-axis; control field and RF field are considered as standing waves and probe field is considered as running field.

Next, the behavior of atom localization is studied considering only control field to be standing wave i.e. $\Omega_{c x y}=\Omega_{c}(\sin k 1 x+\sin k 2 y)$ and the result is shown in Fig. 4. The probe, control and RF Rabi frequencies are kept same as were in Fig. 2. A significant change has been observed in the localization pattern. The finding probability of an atom now restricts to only two quadrants i.e. second and fourth quadrants with the maxima of the probe absorption still distributed diagonally. Other system parameters are same as were in Fig. 3.

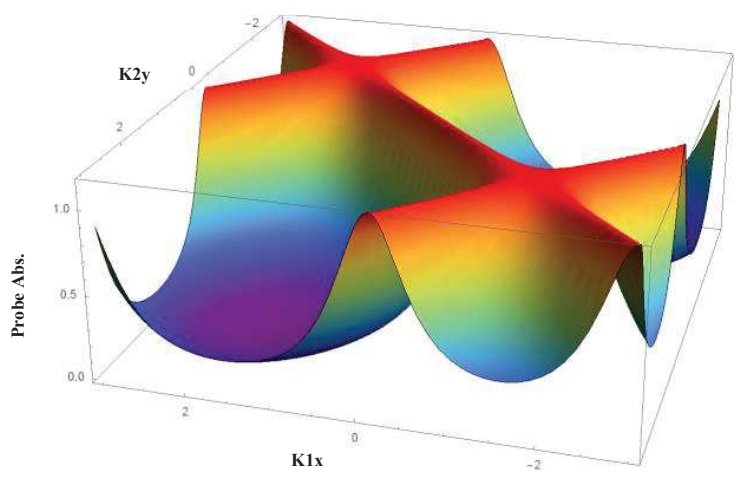

Figure 4 Probe absorption as a function of wave vectors $\mathrm{k}_{1} \mathrm{x}$ and $\mathrm{k}_{2} \mathrm{y}$ reflecting the position probability as crater like structures in second as fourth quadrant; control field is considered as standing wave with and probe and RF field as running fields.
This shows if the reflections of control laser is controlled in such a manner that a standing wave is created inside the cavity, the precision in finding the position of an atom may be confined to a smaller region. Further, when RF field is considered to be a combination of standing waves i.e. $\Omega_{r f x y}=\Omega_{r f}(\sin k 1 x+\sin k 2 y)$ by keeping other fields as running waves, the maxima of probe absorption shifts to the alternate quadrants. The atom is now confined to two quadrants with equal probability of finding along with equal degrees of localization in first and third quadrants.

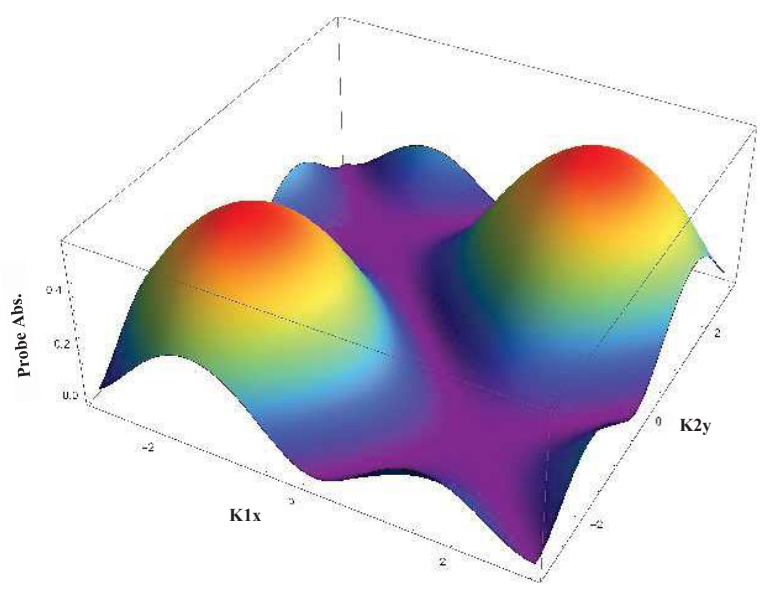

Figure 5 Probe absorption as a function of wave vectors $\mathrm{k}_{1} \mathrm{x}$ and $\mathrm{k}_{2} \mathrm{y}$ reflecting the position probability as bell like structures in first as third quadrant; RF field is considered as standing wave with probe and control fields as running fields.

The results discussed in this work expresses the fact that if propagation and other characteristics of the radiofrequency used in inducing EIT in the system is controlled in such a way that a standing wave is created inside a microwave cavity with atomic vapor cell kept inside interacting with other fields simultaneously, the probability of finding an atom in 2D subwavelength may reach unity. More details about the necessary electromagnetic conditions required to observe the atom localization can be explored by plotting the 3D localization graphs of an atom and is the future plan of our group.

\section{Conclusion}

The behavior of $2 \mathrm{D}$ atom localization is explored in this work. We studied the behavior of probe absorption in three different cases. First, when both control and RF fields are considered as standing waves, the pattern is observed as a running wave and atom cannot be localized precisely. Second, when only control field is considered as standing wave the atom is more confined as compared to the previous result and is localized in the second and fourth quadrant, but the maxima are still distributed. At last, when RF field is considered as standing wave the atom is localized in first and third quadrant with equal 
probability of finding the atom in both the quadrants. More investigation is required in this study to precisely understand the parameters governing the localization of an atom. Radio-frequency E-field metrology may utilize the concept of 2D and 3D atom localization for vector Efield measurements with minimum uncertainty component.

\section{References}

1. E. Arimondo, "V coherent population trapping in laser spectroscopy," Progress in Optics. 35, 1996, pp. 257-354, doi: 10.1016/S0079-6638(08)70531-6.

2. U. Fano, "Effects of configuration interaction on intensities and phase shifts,". Physical Review, 124, 6, December 1961, pp. 1866-78, doi: 10.1103/PhysRev.124.1866.

3. L. A. Lugiato, "II theory of optical bistability," Progress in optics, 21, 1984, pp. 69-216, doi: $10.1016 / \mathrm{S} 0079-6638(08) 70122-7$.

4. L. V. Hau, S. E. Harris, Z. Dutton, and C. H. Behroozi, "Light speed reduction to 17 metres per second in an ultracold atomic gas," Nature, 397, 1999, pp. 594-598.

5. S. E. Harris, J. E. Field, and A. Imamoğlu, "Nonlinear optical processes using electromagnetically induced transparency," Physical Review Letters, 64, 10, 1990, pp. 1107-10, doi: 10.1103/PhysRevLett.64.1107.

6. M. Fleischhauer, A. Imamoglu, and J. P. Marangos, “ Electromagnetically induced transparency: Optics in coherent media," Reviews of modern physics, 77, 2, 2015, pp. 633-73, doi: 10.1103/RevModPhys.77.633.

7. S. H. Autler, and C. H. Townes, "Stark effect in rapidly varying fields," Physical Review, 100, 2, 1955, pp. 703-22, doi: 10.1103/PhysRev.100.703.

8. H. Fan, S. Kumar, J. Sedlacek, H. Kübler, S. Karimkashi, \& J. P. Shaffer, "Atom based RF electric field sensing," Journal of Physics B: Atomic, Molecular and Optical Physics, 48, 20, September 2015, p. 16, doi: 10.1088/0953-4075/48/20/202001.

9. C. L. Holloway, J. A. Gordon, S. Jefferts, A. Schwarzkopf, D. A. Anderson, S. A. Miller, N. Thaicharoen, and G. Raithel, "Broadband Rydberg Atom-Based Electric-Field Probe for SI-Tracable, Self-Calibrated Measurements," IEEE Transactions on Antennas and Propagation, 62, 12, December 2014, pp. 6169-82, doi: 10.1109/TAP.2014.2360208.

10. C. L. Holloway, M. T. Simons, J. A. Gordon, A. Dienstfrey, D. A. Anderson, and G. Raithel, "Electric Field Metrology for SI Traceability: Systematic Measurement Uncertainties in Electromagnetically
Induced Transparency in Atomic Vapor," Journal of Applied Physics, 121, 23, June 2017, pp. 9, doi: https://doi.org/10.1063/1.4984201.

11. J. A. Sedlacek, A. Schwettmann, H. Kubler, R. Low, T. Pfau, and J. P. Shaffer, "Microwave Electrometry with Rydberg Atoms in a Vapour Cell Using Bright Atomic Resonances," Nature Physics, 8, November 2012, pp. 819-24. Doi: 10.1038/NPHYS2423.

12. Li, H., Sautenkov et al, "Optical imaging beyond the diffraction limit via dark states," Physical Review A, 78, 1, July 2008, pp. 013803-1-6. doi: 10.1103/PhysRevA.78.013803.

13. J. A. Miles, Z. J. Simmons, and D. D. Yavuz, "Subwavelength localization of atomic excitation using electromagnetically induced transparency," Physical Review X, 3, 3, September 2013, pp. 031014-1-8, doi: 10.1103/PhysRevX.3.031014.

14. J. R. Gardner, M. L. Marable, G. R. Welch, and J. E. Thomas, "Suboptical wavelength position measurement of moving atoms using optical fields," Physical review letters, 70, 22, May 1993, pp. 3404-07, doi: 10.1103/PhysRevLett.70.3404.

15. C. Ding, J. Li, X. Yang, D. Zhang, and H. Xiong, "Proposal for efficient two-dimensional atom localization using probe absorption in a microwavedriven four-level atomic system," Physical Review A, 84, 4, October 2011, pp. 043840-1-9, doi: 10.1103/PhysRevA.84.043840.

16. H. R. Hamedi, and G. Juzeliūnas, "Phase-sensitive atom localization for closed-loop quantum systems," Physical Review A, 94, 1, July 2016, pp. 013842-1-11, doi: 10.1103/PhysRevA.94.013842.

17. N. Singh, and A. Wasan, "High-precision two-and three-dimensional atom localization via spatial dependent probe absorption in a closed-loop M-type atomic system," Journal of the Optical Society of America B, 35, 6, May 2018, pp. 1318-27, doi: 10.1364/JOSAB.35.001318.

18. C. Zhu, C. Tan, and G. Huang, "Crossover from electromagnetically induced transparency to AutlerTownes splitting in open V-type molecular systems," Physical Review A, 87, 4, April 2013, pp. 043813-1-13, doi: 10.1103/PhysRevA.87.043813. 